\title{
ON THE DIMENSION OF CHOWLA-MILNOR SPACE
}

\author{
TAPAS CHATTERJEE
}

\begin{abstract}
In a recent work, Gun, Murty and Rath defined the ChowlaMilnor space and proved a non-trivial lower bound for these spaces. They also obtained a conditional improvement of this lower bound and noted that an unconditional improvement of their lower bound will lead to irrationality of $\zeta(k) / \pi^{k}$ for odd positive integers $k>1$. In this paper, we give an alternate proof of their theorem about the conditional lower bound.
\end{abstract}

\section{INTRODUCTION}

For any complex number $s \in \mathbb{C}$, with $\Re(s)>1$, one defines the Riemann zeta function as

$$
\zeta(s)=\sum_{n=1}^{\infty} \frac{1}{n^{s}}
$$

which has an Euler product

$$
\zeta(s)=\prod_{p}\left(1-p^{-s}\right)^{-1} .
$$

The Riemann zeta function defines an analytic function in the region $\Re(s)>1$ and can be extended meromorphically to the whole complex plane with a simple pole at $s=1$ having residue 1 . Hurwitz generalized the Riemann zeta function by $\zeta(s, x)$ which is defined as

$$
\zeta(s, x)=\sum_{n=0}^{\infty} \frac{1}{(n+x)^{s}}
$$

where $0<x \leq 1$ and $s \in \mathbb{C}$ with $\Re(s)>1$. He proved that $\zeta(s, x)$ can be extended meromorphically to the entire complex plane with a pole at $s=1$. Note that for $x=1, \zeta(s, 1)$ is the classical Riemann zeta function.

Definition. For integers $k>1, q \geq 2$, define the Chowla- Milnor space $V_{k}(q)$ by

$$
V_{k}(q):=\mathbb{Q}-\operatorname{span} \text { of }\{\zeta(k, a / q): 1 \leq a<q,(a, q)=1\} .
$$


As described in [1], the conjecture of Chowla and Milnor is the assertion that the dimension of $V_{k}(q)$ is equal to $\varphi(q)$, where $\varphi$ is the Euler's phifunction. Gun, Murty and Rath [1] show that the dimension of the above spaces is at least $\varphi(q) / 2$. They also derived the following theorem.

Theorem. Let $k>1$ be an odd integer and $q, r>2$ be two co-prime integers. Then either

or

$$
\operatorname{dim}_{\mathbb{Q}} V_{k}(q) \geq \frac{\varphi(q)}{2}+1
$$

$$
\operatorname{dim}_{\mathbb{Q}} V_{k}(r) \geq \frac{\varphi(r)}{2}+1 .
$$

The proof in [1] uses the expansion of Bernoulli polynomials. In this note, we give an alternate proof of the theorem by an explicit evaluation of co-tangent derivatives.

\section{Proof of the Theorem}

The following lemma 1 due to Okada [2] about the linear independence of co-tangent values at rational arguments plays a significant role in proving the theorem.

Lemma 1. Let $k$ and $q$ be positive integers with $k \geq 1$ and $q>2$. Let $\mathrm{T}$ be a set of $\varphi(q) / 2$ representations $\bmod q$ such that the union $T \cup(-T)$ constitutes a complete set of co-prime residue classes mod $q$. Then the set of real numbers

$$
\left.\frac{d^{k-1}}{d z^{k-1}} \cot (\pi z)\right|_{z=a / q}, \quad a \in T
$$

are linearly independent over $\mathbb{Q}$.

We first have the following lemma.

Lemma 2. For an integer $k \geq 1$,

$$
D^{k-1}(\pi \cot \pi z)=\pi^{k} \times \mathbb{Z} \text { linear combination of }(\csc \pi z)^{2 l}(\cot \pi z)^{k-2 l},
$$

for some non-negative integer $l$. Here $D^{k-1}=\frac{d^{k-1}}{d z^{k-1}}$.

Proof. We will prove this by induction on $k$. For $k=1$, we have

$D^{k-1}(\pi \cot (\pi z))=\pi \cot (\pi z)$. Assume that the statement is true for $k-1$, i.e.

$$
D^{k-2}(\pi \cot (\pi z))=\pi^{k-1} \sum a_{i}(\csc \pi z)^{2 l_{i}}(\cot \pi z)^{(k-1)-2 l_{i}}
$$

where $a_{i}$ 's are integers.

Differentiating both sides with respect to $z$ we get,

$$
\begin{aligned}
D^{k-1}(\pi \cot \pi z)=\pi^{k} \sum & {\left[b_{i}(\csc \pi z)^{2 l_{i}}(\cot \pi z)^{k-2 l_{i}}\right.} \\
& \left.+c_{i}(\csc \pi z)^{2 l_{i}+2}(\cot \pi z)^{k-\left(2 l_{i}+2\right)}\right]
\end{aligned}
$$


where $b_{i}, c_{i}$ 's are integers. This completes the proof of lemma 2 .

Lemma 3. For an integer $k \geq 2$,

$$
\zeta(k, a / q)+(-1)^{k} \zeta(k, 1-a / q)=\left.\frac{(-1)^{k-1}}{(k-1) !} D^{k-1}(\pi \cot \pi z)\right|_{z=a / q} .
$$

\section{Proof.}

$$
\begin{aligned}
\text { L.H.S. } & =\zeta(k, a / q)+(-1)^{k} \zeta(k, 1-a / q) \\
& =\sum_{n \geq 0}^{\infty} \frac{1}{(n+a / q)^{k}}+(-1)^{k} \sum_{n \geq 0}^{\infty} \frac{1}{(n+1-a / q)^{k}} \\
& =\sum_{n \geq 0}^{\infty} \frac{1}{(n+a / q)^{k}}+(-1)^{k} \sum_{n=1}^{\infty} \frac{1}{(n-a / q)^{k}} \\
& =\sum_{n \geq 0}^{\infty} \frac{1}{(n+a / q)^{k}}+(-1)^{2 k} \sum_{n=1}^{\infty} \frac{1}{(-n+a / q)^{k}} \\
& =\sum_{n \in \mathbb{Z}} \frac{1}{(n+a / q)^{k}} .
\end{aligned}
$$

Again we know that for $z \notin \mathbb{Z}$,

$$
\pi \cot \pi z=\sum_{n \in \mathbb{Z}} \frac{1}{z+n} .
$$

This implies that

$$
D^{k-1}(\pi \cot \pi z)=(-1)^{k-1}(k-1) ! \sum_{n \in \mathbb{Z}} \frac{1}{(z+n)^{k}} .
$$

So,

$$
\left.\frac{(-1)^{k-1}}{(k-1) !} D^{k-1}(\pi \cot \pi z)\right|_{z=a / q}=\sum_{n \in \mathbb{Z}} \frac{1}{(n+a / q)^{k}},
$$

which completes the proof of lemma 3.

Finally, we have lemma 4, whose proof is standard.

Lemma 4. Let $\mathrm{P}$ be the set of primes. We have

$$
\zeta(k) \prod_{\substack{p \in \mathrm{P}, p \mid q}}\left(1-p^{-k}\right)=q^{-k} \sum_{\substack{a=1 \\(a, q)=1}}^{q-1} \zeta(k, a / q) .
$$


Proof of the Theorem. First note that the space $V_{k}(q)$ is also spanned by the following sets of real numbers:

$$
\begin{aligned}
& \{\zeta(k, a / q)+\zeta(k, 1-a / q) \mid(a, q)=1,1 \leq a<q / 2\}, \\
& \{\zeta(k, a / q)-\zeta(k, 1-a / q) \mid(a, q)=1,1 \leq a<q / 2\} .
\end{aligned}
$$

Now from the lemma 3 , we have the following

$$
\zeta(k, a / q)+(-1)^{k} \zeta(k, 1-a / q)=\left.\frac{(-1)^{k-1}}{(k-1) !} D^{k-1}(\pi \cot \pi z)\right|_{z=a / q} .
$$

Applying the above lemma 1, we see that

$$
\operatorname{dim}_{\mathbb{Q}} V_{k}(q) \geq \frac{\varphi(q)}{2} .
$$

Now from lemma 2 and lemma 3 for an odd integer $k$, we have

$$
\begin{aligned}
& \frac{\zeta(k, a / q)-\zeta(k, 1-a / q)}{(2 \pi i)^{k}} \\
& =\frac{i}{2^{k}} \times \mathbb{Q} \text { linear combinations of }(\csc \pi a / q)^{2 l}(\cot \pi a / q)^{k-2 l}
\end{aligned}
$$

We note that

$$
i \cot (\pi a / q)=\frac{1+\zeta_{q}^{a}}{1-\zeta_{q}^{a}}
$$

belongs to $\mathbb{Q}\left(\zeta_{q}\right)$ and hence so do the numbers $\csc (\pi a / q)^{2 l}$ and $\cot (\pi a / q)^{2 l}$. Since $k$ is odd, we have

$$
\frac{\zeta(k, a / q)-\zeta(k, 1-a / q)}{(2 \pi i)^{k}} \in \mathbb{Q}\left(\zeta_{q}\right)
$$

Now we go back to the main part of the proof. Let $q$ and $r$ be two co-prime integers. Suppose that

Then the numbers

$$
\operatorname{dim}_{\mathbb{Q}} V_{k}(q)=\frac{\varphi(q)}{2}
$$

$$
\zeta(k, a / q)-\zeta(k, 1-a / q), \text { where }(a, q)=1,1 \leq a<q / 2
$$

generate $V_{k}(q)$. Now from lemma 4 , we get

$$
\zeta(k) \prod_{p \mid q}\left(1-p^{-k}\right)=q^{-k} \sum_{\substack{a=1,(a, q)=1}}^{q-1} \zeta(k, a / q) \in V_{k}(q) .
$$

and hence

$$
\zeta(k)=\sum_{\substack{(a, q)=1 \\ 1 \leq a<q / 2}} \lambda_{a}[\zeta(k, a / q)-\zeta(k, 1-a / q)], \lambda_{a} \in \mathbb{Q}
$$


so that

$$
\frac{\zeta(k)}{(2 \pi i)^{k}}=\sum_{\substack{(a, q)=1 \\ 1 \leq a<q / 2}} \frac{\lambda_{a}[\zeta(k, a / q)-\zeta(k, 1-a / q)]}{(2 \pi i)^{k}}
$$

Thus by (11)

Similarly , if

$$
\frac{\zeta(k)}{i \pi^{k}} \in \mathbb{Q}\left(\zeta_{q}\right)
$$

then

$$
\operatorname{dim}_{\mathbb{Q}} V_{k}(r)=\frac{\varphi(r)}{2}
$$

and hence

$$
\frac{\zeta(k)}{i \pi^{k}} \in \mathbb{Q}\left(\zeta_{r}\right)
$$

$$
\frac{\zeta(k)}{i \pi^{k}} \in \mathbb{Q}\left(\zeta_{q}\right) \cap \mathbb{Q}\left(\zeta_{r}\right) .
$$

Since any non-trivial finite extension of $\mathbb{Q}$ is ramified, if $\mathbb{Q}\left(\zeta_{q}\right) \cap \mathbb{Q}\left(\zeta_{r}\right) \neq \mathbb{Q}$ then there exists a prime which is ramified in $\mathbb{Q}\left(\zeta_{q}\right) \cap \mathbb{Q}\left(\zeta_{r}\right)$, hence both in $\mathbb{Q}\left(\zeta_{q}\right)$ and $\mathbb{Q}\left(\zeta_{r}\right)$. Note a prime which ramifies in this intersection must necessarily divide both $q$ and $r$. This is impossible because $(q, r)=1$. So $\mathbb{Q}\left(\zeta_{q}\right) \cap \mathbb{Q}\left(\zeta_{r}\right)=\mathbb{Q}$. Hence we arrive at a contradiction as $\frac{\zeta(k)}{\pi^{k}}$ is a real number. Thus

$$
\operatorname{dim}_{\mathbb{Q}} V_{k}(q) \geq \frac{\varphi(q)}{2}+1 \quad \text { or } \quad \operatorname{dim}_{\mathbb{Q}} V_{k}(r) \geq \frac{\varphi(r)}{2}+1 .
$$

This completes the proof of the theorem.

Acknowledgement. I would like to thank Sanoli Gun for helpful discussions.

\section{REFERENCES}

[1] S. Gun, M. Ram Murty and P. Rath, On a conjecture of Chowla and Milnor, Canadian J. Math. 63(6),2011, 1328-1344.

[2] T. Okada, On an extension of a theorem of S. Chowla, Acta Arith. 38 (1980/81), no. $4,341-345$.

(Tapas Chatterjee) The Institute of Mathematical Sciences, Cit Campus, Taramani, Chennai 600113 India

E-mail address, Tapas Chatterjee: tapasc@imsc.res.in 